\title{
HIP ARTHRITIS AS A CLINICAL PRESENTATION OF BONE LYMPHOMA
}

\author{
Marcella Azevedo Borges Andrade ${ }^{1, *}$, Clarissa Canella², Flávia Martins Costa², Marcelo Bragança dos Reis Oliveira ${ }^{1}$, Blanca \\ Elena Rios Gomes Bica
}

1.Universidade Federal do Rio de Janeiro, Rio de Janeiro (RJ), Brazil. 2.Centro de Diagnóstico por Imagem, Rio de Janeiro (RJ), Brazil.

*Corresponding author: marcella.reumato@gmail.com

\section{BACKGROUND}

Musculoskeletal complaints in patients with non-Hodgkin's lymphoma (NHL) are not unusual, but peripheral joint involvement as a presenting feature is quite rare.

\section{CASE REPORT}

A 31-year-old man, previously healthy, was evaluated by a rheumatologist for pulsatile pain in the right hip with irradiation to the anterolateral thigh region. The patient had been well until 2 years before this evaluation, when the joint pain started. Six months before the presentation, the pain was more severe (VAS 8), continuous, with functional limitation, difficulty to sleep because of the pain and without improvement with the use of common analgesics or nonsteroidal anti-inflammatory drugs. He denied trauma, pain in the spine or other peripheral joints, fever, weight loss. On examination, he presented pain with motion of the right hip, without signs of enthesitis, normal Schober, without other changes. Magnetic resonance imaging (MRI) of the pelvis showed hip joint effusion with slight post-contrast enhancement, suggesting synovitis; MRI of sacroiliac joints using the STIR technique did not show sacroiliitis or enthesitis. Laboratory tests showed increased C-reactive protein level ( $0.73 \mathrm{mg} / \mathrm{dL}$ ); negative autoantibodies (ANA, rheumatoid factor, anti-CCP, anti-Ro, anti-La, anti-RNP, anti-Sm); HLA B27 negative; normal beta-2-microglobulin, renal function, liver function, blood count and serum protein electrophoresis. Opted to perform MRI of the right thigh (Figs. 1-3), which visualized an irregular and heterogeneous hyperintense image of the bone marrow of the right femoral diaphysis, measuring $22 \mathrm{~cm}$ longitudinally, associated with marked irregularity, circumferential cortical thickening and small areas of periosteal reaction; extension of the lesion to the medial soft tissues; elongated image with restricted diffusion and peripheral contrast enhancement, measuring $2.7 \mathrm{~cm}$, indicative of collection. The radiological impression was of a heterogeneous lytic lesion in the femoral diaphysis, associated to a soft tissue's collection with solid component, which could be considered a possibility of Ewing's carcinoma or chronic osteomyelitis. The patient underwent bone biopsy of the right femur, whose histopathological and immunohistochemical report was of high-grade non-Hodgkin's B lymphoma infiltrating periosteal connective tissue. A whole-body PET-CT was not positive in another site than the right thigh. Chemotherapy treatment started, with good response.

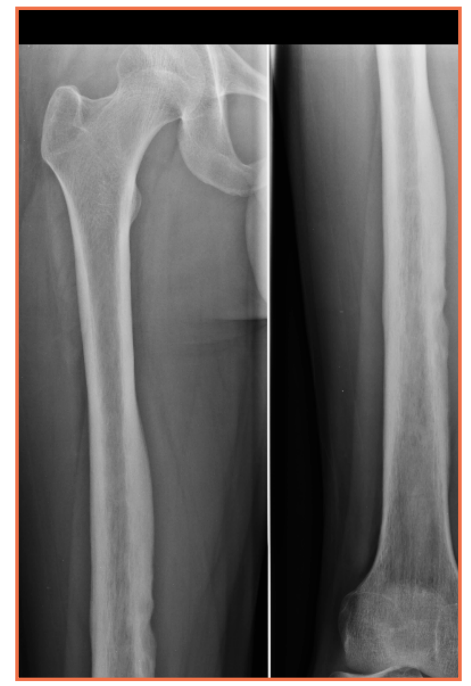

Figure 1. X-ray of the thigh showing solid and regular cortical thickening of the medial aspect of the diaphysis. 


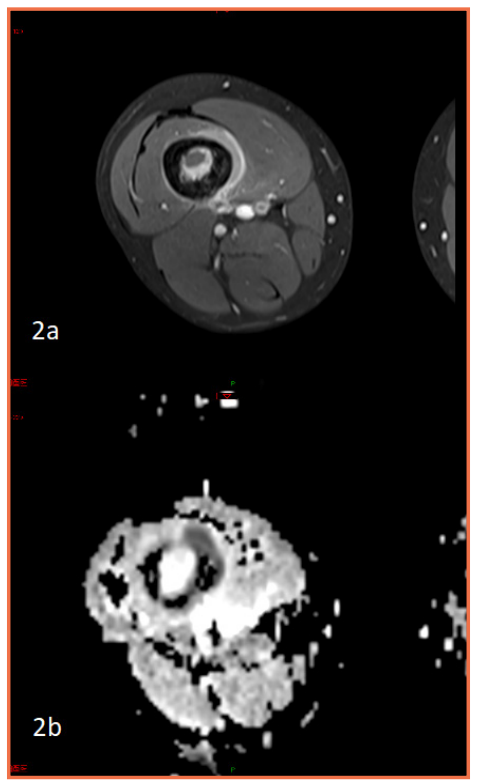

Figure 2. Thigh on axial plane weighted on T1 fat saturation after gadolinium administration (a) showing juxtacortical lesion with peripheral enhancement presenting restricted diffusion on DWI images (b) suggesting tumoral lesion;
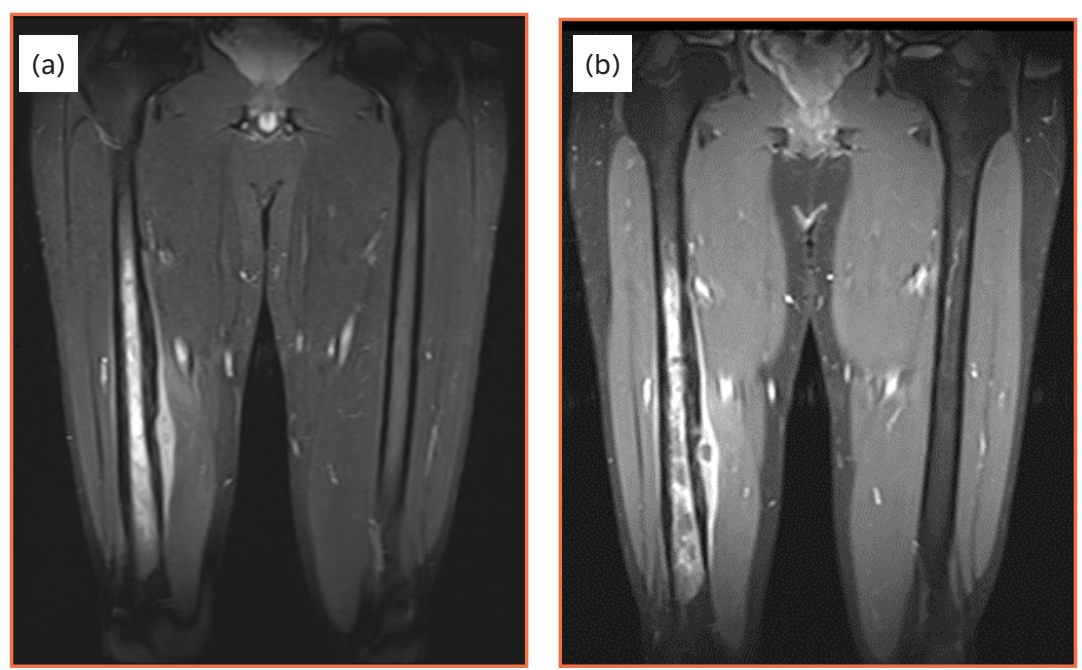

Figure 3. Thigh on coronal plane weighted on T1 fat saturation after gadolinium administration (a) and STIR sequences (b) showing juxtacortical lesion with peripheral enhancement and soft tissue edema. Note also the edema of the adjacent bone marrow.

\section{CONCLUSION}

Skeletal involvement is described in $25 \%$ of patients with non-Hodgkin's lymphoma, typically as secondary, rarely as primary lymphoma of bone. Moreover, these patients may present with monoarticular or polyarticular arthritis. It is essential that the rheumatologist be alert during the investigation of pain, as serious diseases can mimic the rheumatic diseases. 